\title{
УДК 004.42
}

\section{MODERN DEVELOPMENT TOOLS FOR THE SERVER SIDE OF} WEBSITES

\section{СОВРЕМЕННЫЕ СРЕДСТВА РАЗРАБОТКИ СЕРВЕРНОЙ ЧАСТИ ШЕВ-САЙТОВ}

Rusakov A.M. / Русаков A.M. senior lecturer / cm. преподаватель. SPIN: 1066-8077

Semykin D.A. / Семыкин Д.А. student / студент.

MIREA-Russian Technological University, Moscow, PR-t Vernadsky, 78 МИРЭА - Российский технологический университет, Москва, Пр-т Вернадского, 78

Аннотация. В статье приводятся основные особенности создания web-caйтов. Описаны основные цели и области применения клиентской и серверной разработки. Рассмотрены современные технологии для серверной разработки: C\#, PHP, Python, Node.js, Јаva, - а также соответствуюшие библиотеки. Показана актуальность технологий в сфере жеb-разработки на рынке труда.

Ключевые слова: web-caŭm, средства разработка серверной части, языки программирования.

\section{Введение}

Каждый человек, хотя бы имеющий смартфон или средство выхода в интернет, сталкивается c web-серверами в повседневной жизни, посещая webсайты или web-приложения. Web-сервер представляет совокупность аппаратных и программных средств, необходимых для обмена запросами с пользователями, при помощи протокола передачи гипертекста - HTТР. Пример такого обращения: пользователь обращается к сайту, а сайт возвращает webстраницу; пользователь нажимает на кнопку для входа в свою социальную сеть, тем самым отправляя на web-cервер запрос, содержащий свой логин и пароль, a в ответ web-сервер может вернуть либо web-страницу пользователя, либо сообщение об ошибке доступа [5].

Взаимодействия клиента и сервера по сети организуются по принципу клиент-серверной модели. Для того, чтобы обратиться к web-cepверу пользователю необходим клиент, таким клиентом выступают web-браузеры (Например: Opera, Firefox, Internet Explorer и т.д.). В связи с этим, разработку web-сайтов разделяют на серверную и клиентскую разработку [1].

\section{Клиентская разработка}

Более популярное название клиентской стороны - фронтенд. Клиент - это то, с чем взаимодействует пользователь. Так что «клиентский»код отвечает за большую часть того, что на самом деле видит пользователь. Разработчики на стороне клиента используют свои навыки программирования для создания визуально привлекательных, функциональных и полезных веб-приложений и динамических веб-сайтов. Эти программисты несут ответственность за каждую часть веб-сайта, которую пользователи видят или с которой взаимодействуют. Домашние страницы, страницы покупок, слайд-шоу - практически любая видимая функция, которая отображается в веб-браузере или требует ввода 
данных пользователем, попадает под эту дисциплину. Всё это программное обеспечение работает на клиентском устройстве. Веб-разработчики не могут контролировать, какой браузер может использовать каждый пользователь для просмотра веб-сайта - браузеры обеспечивают противоречивые уровни совместимости с функциями кода на стороне клиента, и одной из задач программирования на стороне клиента является изящная обработка различий в поддержке браузера. Общие языки сценариев, используемые разработчиками интерфейса для создания клиентского кода, включают JavaScript, HTML и CSS.

\section{Серверная разработка}

Более популярное название серверной стороны - бэкенд. Сервер в вебприложении прослушивает запросы, поступающие от клиента. Код на стороне сервера обрабатывает такие задачи, как проверка отправленных данных и запросов, использование баз данных для хранения и извлечения данных и отправка правильных данных клиенту по мере необходимости. Код серверной части может быть написан на любом количестве языков программирования примеры популярных языков серверной части включают в себя РНР, Python, Ruby, C\# и NodeJS (JavaScript) [2]. Код серверной части имеет полный доступ к операционной системе сервера, и разработчик может выбрать какой язык программирования (и какую версию) он хотел бы использовать.

Программирование серверной части очень полезно поскольку позволяет эффективно доставлять информацию, составленную для индивидуальных пользователей и, таким образом, создавать намного лучший опыт использования [6].

Компании, такие как Amazon, используют программирование серверной части для построения исследовательских результатов для товаров, формирования целевого предложения, основанного на предпочтениях клиента и предыдущих покупках, упрощения заказов и т. д. Банки используют программирование серверной части, чтобы хранить учётную информацию и позволять только авторизованным пользователям просматривать и совершать транзакции. Другие сервисы, такие как Facebook, Twitter, Instagram и Wikipedia используют бэкенд, чтобы выделять, распространять и контролировать доступ к интересному контенту.

Разработчики обычно пишут свой код, используя веб-фреймворки. Вебфреймворки - это наборы функций, объектов, правил и других конструкций кода, предназначенных для решения общих проблем, ускорения разработки и упрощения различных типов задач, стоящих в конкретной области.

И снова, поскольку и клиентская и серверная части используют фреймворки, области очень разные и, следовательно, фреймворки тоже разные. Фреймворки клиентской части упрощают вёрстку и представление данных, в то время как фреймворки серверной части обеспечивают много «обычной» функциональности веб-сервера, котору, возможно, в противном случае, пришлось бы осуществлять самостоятельно (например, поддержка сессий, поддержка пользователей и аутентификация, простой доступ к базе данных, шаблонам библиотек и т. д.). 


\section{Цели применения бэкенда:}

1. Эффективное хранение и доставка информации.

Например, Amazon содержит большое количество товаров, создание статической страницы для каждого товара или поста было бы абсолютно неэффективным. Программирование серверной части позволяет вместо этого хранить информацию в базе данных и динамически создавать и возвращать HTML и другие типы файлов (например, PDF, изображения, и т. д.). Также есть возможность просто вернуть данные (JSON, XML, и т. д.) для отображения, используя подходящий фреймворк клиентской части (это уменьшает загрузку процессора на сервере и количество передаваемых данных).

Сервер не ограничен в отправке информации из баз данных и может вместо этого возвращать результат инструментов программного обеспечения или данные из сервисов коммуникации. Контент даже может быть целевым относительно устройства клиента, который его получает.

Из-за того, что информация находится в базе данных, её также можно легко передать и обновить через другие бизнес системы (например, отслеживание).

\section{2. Настраиваемый пользовательский опьт взаимодействия.}

Серверы могут хранить и использовать информацию о клиентах чтобы поставлять удобный и сделанный индивидуально пользовательский опыт взаимодействия. Например, многие сайты хранят данные кредитных карт, чтобы не нужно было вводить их повторно. Сайты, наподобие Google Maps, могут использовать сохранённое и текущее местоположение для предоставления информации о маршруте, а также историю поиска или путешествий для выделения местных предприятий в результатах поиска. Более глубокий анализ привычек пользователя может быть использован для прогнозирования их интересов и дальнейших настроек ответов и уведомлений, например, предоставление списка ранее посещённых популярных мест, которые вы, возможно, захотите найти на карте.

3. Контролируемый доступ к контенту.

Программирование серверной части позволяет сайтам ограничивать доступ авторизованным пользователям и предоставлять только ту информацию, которую пользователю разрешено видеть.

Например, социальные сети, такие как Facebook, позволяют пользователям полностью контролировать свои данные, но только своим друзьям разрешать просматривать или комментировать их. Пользователь определяет, кто может просматривать его данные и, более того, чьи данные появляются на его стене. Авторизация - центральная часть опыта взаимодействия.

4. Хранение информащии о сессии/состоянии.

Программирование серверной части позволяет разработчикам использовать сессии - изначально это механизм, позволяющий серверу хранить информацию о текущем пользователе сайта и отправлять разные ответы, основанные на этой информации.

Это позволяет, например, сайту знать, что пользователь был предварительно авторизован и выводить ссылки на его адрес электронной 
почты или историю заказов или, возможно, сохранить прогресс простой игры, так чтобы пользователь мог вернуться на сайт продолжить с того места, где он закончил.

5. Уведомления и средства связи.

Серверы могут отправлять общие или пользовательские уведомления непосредственно через сайт или по электронной почте, через смс, мгновенные сообщения, видеосвязь или другие средства связи.

Самый распространённый вид уведомлений - это «подтверждение регистрации». Часто при создании новой учётной записи на таких крупных сайтах, как Google, Amazon, Instagram и т. п., пользователь вскоре получает письмо на свою указанную при регистрации, подтверждающее факт регистрации или содержащее информацию о необходимости активировать учётную запись.

\section{6. Анализ данных .}

Веб-сайт может собирать много данных о своих пользователях: что они ищут, что они покупают, что они рекомендуют, как долго они остаются на каждой странице. Программирование серверной части может быть использовано, чтобы усовершенствовать ответы, основанные на анализе этих данных.

Например, и Amazon, и Google рекламируют товары на основании предыдущих поисков (и покупок) [7].

\section{Анализ средств разработки готовых web-страниц в сети}

Для чтобы проверить, при помощи каких средств реализован web-cайт, может каждый воспользовавшись расширением для Google Chrome Wappalyzer. Он обнаруживает системы управления контентом, веб-магазины, веб-серверы, JavaScript-фреймворки, аналитические инструменты и многое другое.

chrome web store

Home > Extensions > Wappalyzer

$\$$ Wappalyzer

Add to Chrome

Offered by: wappalyzer.com

$\star \star \star \star \star 1,724 \mid$ Developer Tools $\mid \doteq 1,054,295$ users

\%

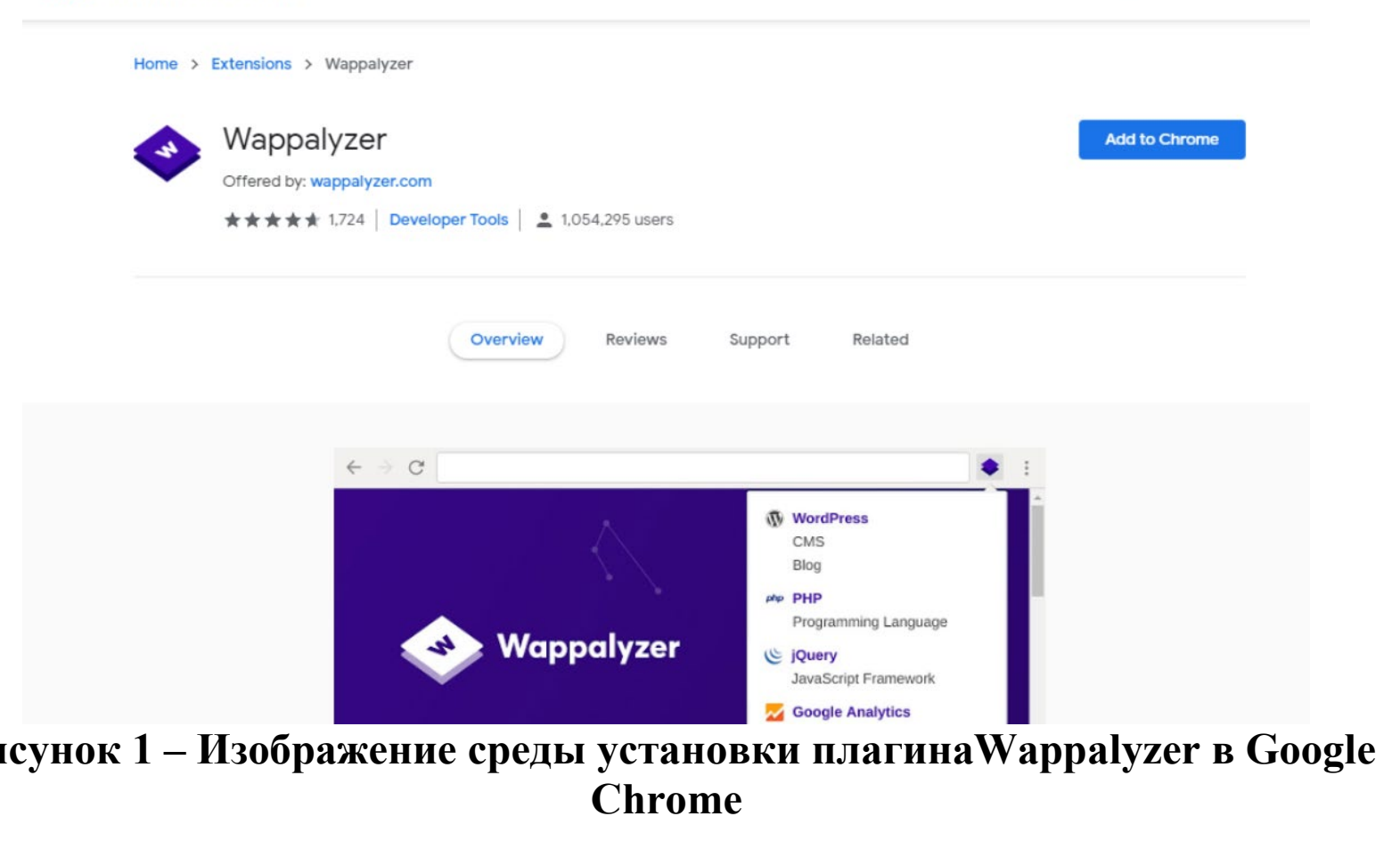


Однако, поскольку серверная разработка располагается не на стороне клиента, зачастую возможностей, предоставляемых расширением, оказывается недостаточно. Тем не менеe, Wappalyzer очень полезен в анализе средств разработки web-страниц.

Для того, чтобы это сделать необходимо только перейти на web-cайт и нажать на иконку расширения, тогда wappalyzer отобразит информацию о используемых технологиях на сайте.

\section{- Wappalyzer}

Аналитика

Ill Yandex.Metrika

JS-фреймворк

- BEM
Прочее

webpack

JS-библиотека

React 17.0 .2

(E) jQuery 2.1.4

\section{Lead generation - CRM integration - API}

\section{Рисунок 2 - Результат проверки сайта https://yandex.ru/ при помощи программного средства Wappalyzer}

\section{Современные средства разработки серверной части web-сайтов Язык программирования - С\#}

Язык программирования С\#- объектно-ориентированный язык программирования. С\# прекрасно подходит для разработки серверных приложений. С\# занимает 4 место на май 2021 года в рейтинге TIOBE [13]. Данный рейтинг позволяет следить за динамикой популярности различных языков программирования, многие факторы влияют на формирование рейтинга, такие как: количество разработчиков в определенной сфере, доступность курсов по конкретным языкам, а также решения, которые позволяют расширить возможности языка. Вместе с фреймворком ASP.NET C\# предоставляет обширное количество классов для разработки web-серверов, позволяет реализовать многопоточность и имеет высокую популярность среди разработчиков. Существуют две версии фреймворков: ASP.NET 5 и ASP.NET Core.

ASP.NET занимает 4 место в разделе "Web Frameworks" в опросе разработчиков портала Stack Overflow за 2020 год [4]. 
Среди web-серверов написанных при помощи этих средств, можно выделить:
1) Microsoft
2) MSN.com

\section{Язык программирования - РНР}

Язык программирования РНР (от англ. препроцессор гипертекста) - язык программирования общего назначения, широко используемый в вебразработке. Рhр достаточно старая технология, но тем не менее, огромное количество сайтов выбирают его, в связи с этим необходимость рhрразработчиков актуальна. Как правило в совокупности применяется web-cepвер Apache или nginx. PHР занимает 9 место в рейтинге TIOBE.

Для разработки бэкенда в phр существуют популярные готовые решения, предоставляемые фреймворками: Laravel, Yii, Symfony [8].

Laravel - это бесплатный опенсорсный РНР-фреймворк, который появился в 2011 году. С тех пор он смог стать тем фреймворком, который чаще всего выбирают веб-разработчики. Одна из главных причин этого заключается в том, что Laravel позволяет разрабатывать сложные веб-приложения проще, быстрее и безопаснее, чем любые другие фреймворки. Многие возможности, например, поддержка API, доступны из коробки. Кроме того, есть много полезных пакетов с дополнительной функциональностью. Основная проблема Laravel недостаточная производительность по сравнению с Django или Express. Для тяжелых проектов это может стать существенным минусом.

Laravel занимает 12 место в разделе "Web Frameworks" в опросе разработчиков портала Stack Overflow за 2020 год.

Yii - это простой, но высокопроизводительный универсальный фреймворк, основанный на компонентной структуре. Он известен благодаря высокой производительности, но, в первую очередь, он знаменит своей простотой. Этот фреймворк появился в декабре 2008 года. Он позволяет использовать код сторонних разработчиков, а имеющийся в нём генератор кода Gii позволяет быстро создавать базовые структуры, на основе которых можно строить собственные решения.

Его создатели очень серьёзно подошли к вопросам безопасности. В частности, в него встроены механизмы хеширования паролей, основанные на bcrypt, и средства шифрования.

Symfony - РНР-фреймворком, выгодно отличающийся от других надёжностью и зрелостью. Этот фреймворк появился давно, в 2005 году, то есть - существует гораздо дольше, чем большинство других рассматриваемых нами инструментов. Он популярен благодаря соответствию веб-стандартам и шаблонам проектирования РНР.

Он включает в себя встроенную систему тестирования и основан на обширном MVC-фреймворке. Это делает его идеальным выбором для крупномасштабных веб-проектов, создаваемых на уровне организаций. Он, в частности, поддерживает работу с различными базами данных.

Symfony занимает 14 место в разделе "Web Frameworks" в опросе разработчиков портала Stack Overflow за 2020 год. 
Пример web-сайтов, применяющий php:
1) Yahoo
2) Wikipedia.org
3) Facebook
4) Baidu
5) https://www.cyberforum.ru/

\section{Программная платформа - Node.js}

Программная платформа Node.js - это опенсорсная кроссплатформенная среда выполнения для JavaScript, которая работает на серверах. С момента выпуска этой платформы в 2009 году она стала чрезвычайно популярной и в наши дни играет весьма важную роль в области веб-разработки. Эта платформа позволяет писать серверный код для динамических веб-страниц и вебприложений, а также для программ командной строки. На сегодняшний день, практически невозможно найти сайт, не применяющий JavaScript в стеке технологий, как показало время, использовать JS на стороне сервера, оказалось хорошим решением [2].

Фреймворк Node.js занимает первое место в наминации "Other Frameworks, Libraries, and Tools" раздела "Web Frameworks" в опросе разработчиков портала Stack Overflow за 2020 год.

Для разработки web-сайтов на Node.js применяется фреймворк - Express.

Express - это минималистичный и гибкий фреймворк веб-приложений Node.js, который предоставляет набор функций для разработки веб- и мобильных приложений [9]. Он существенно упрощает разработку вебприложений на базе Node. В его возможности входит:

1. Настроить посредников для ответа на запросы НТТР.

2. Определение таблицы маршрутизации, которая используется для выполнения различных действий на основе метода HTTP и URLадреса.

3. Динамическое создание HTML-страниц на основе передачи аргументов шаблонам.

Express занимает 5 место в разделе "Web Frameworks" в опросе разработчиков портала Stack Overflow за 2020 год.

Пример web-сайтов, применяющий Node.js:

1) https://www.mos.ru/

2) Paypal

3) Netflix

4) Uber

\section{Язык программирования - Pуthon}

Язык программирования Python - это скриптовый язык программирования. Он универсален, поэтому подходит для решения разнообразных задач и многих платформ, начиная c iOS и Android и заканчивая серверными ОС. На сегодняшний день python - один из самых популярных языков программирования, обладающий высокой степенью универсальности. Занимает первое место в рейтинге популярности среди остальных языков программирования PYPL за май 2021 года [12]. PYPL (PopularitY of 
Programming Language) - специализированный рейтинг, основанный на данных Google SE. Рейтинг основывается на количестве запросов в Google. B рейтинге TIOВЕ занимает второе место.

Для web-разработки на Python применяется фреймворк - Django.

Django - это популярный, мощный фреймворк на языке Python. Django появился в 2005 году, и постепенно стал одним из лучших фреймворков, который помогал и помогает тысячам разработчиков выполнять ту или иную работу в течение нескольких минут. Фреймворк Django справляется с большим количеством задач и повышенными нагрузками. Его применяют для создания:

- CRM-систем.

- CMS.

- Коммуникационных платформ.

- Сервисов бронирования номеров.

- Платформ управления документооборотом.

Также Django подходит для создания алгоритмических генераторов, платформ для электронных рассылок, систем верификации, систем фильтрации с динамическими правилами и сложными параметрами, платформ для анализа данных и сложных вычислений, машинного обучения.

Django занимает 10 место в разделе "Web Frameworks" в опросе разработчиков портала Stack Overflow за 2020 год.

Пример web-сайтов, применяющий Python:

1) Instagram

2) Google

3) Reddit

4) Spotify

\section{Язык программирования - Јаvа}

Язык программирования Јava - универсальный объектно-ориентированный язык со строгой типизацией $[3,10]$. В нём реализован принцип WORA (от английского: write once, run anywhere). Это позволяет запускать приложения везде, где есть среда исполнения JRE (от английского: Java Runtime Environment). В рейтинге PYPL занимает второе место. В рейтинге TIOBE занимает третье место.

Фреймворк Spring - один из самых популярных фреймворков для создания веб-приложений на Java. Его можно рассматривать как фреймворк фреймворков, поскольку он предлагает поддержку различных фреймворков, таких как Hibernate, Struts, Tapestry, JSF и т. д. Для создания web-сайтов применяется Spring MVC. Он позволяет создавать веб-сайты или RESTful сервисы (например, JSON/XML) и хорошо интегрируется в экосистему Spring, например, он поддерживает контроллеры и REST контроллеры в Spring Boot приложениях. MVC предоставляет архитектуру модель-представлениеконтроллер и готовые компоненты, которые можно использовать для разработки гибких и слабо связанных веб-приложений. Шаблон MVC приводит к разделению различных аспектов приложения (логика ввода, бизнес-логика и логика пользовательского интерфейса), обеспечивая при этом слабую связь между этими элементами. 
Spring занимает 8 место в разделе "Web Frameworks" в опросе разработчиков портала Stack Overflow за 2020 год.

Пример web-сайтов, применяющий Java:

1) Netflix - компания использует Java для серверной части большинства своих приложений, наряду с Python.

2) Spotify - в основном, использует Јava и Python для реализации некоторого функционала своего веб приложения, например для логирования и стабилизации передачи данных. Также Јava является основной технологией для приложения Spotify на Android OS.

3) Amazon - используют Java для разработки своих веб-сервисов (AWS). Они также предпочитают писать на Јava сценарии автоматизации, так как они хорошо поддерживаются, имеют много внутренних инструментов/сред, которые помогают писать вспомогательные инструменты.

Для разработки web-сайтов на Java существует фреймворк Spring.

Актуальность технологий в сфере web-разработки на рынке труда

Для того, чтобы оценить актуальность технологии использовались данные предоставляемые web-сайтом https://hh.ru, как ведущим сервисом по поиску работы в Российской Федерации [11].

В течении двух недель с целью обзора актуальности технологий в области "web" осуществлялся ежедневный поиск по запросам: "C\# web", "Php web", "Node.js Web", "Python web", "Java web". В результате чего, по этим данным были составлены следующие графики.

\section{C\#}

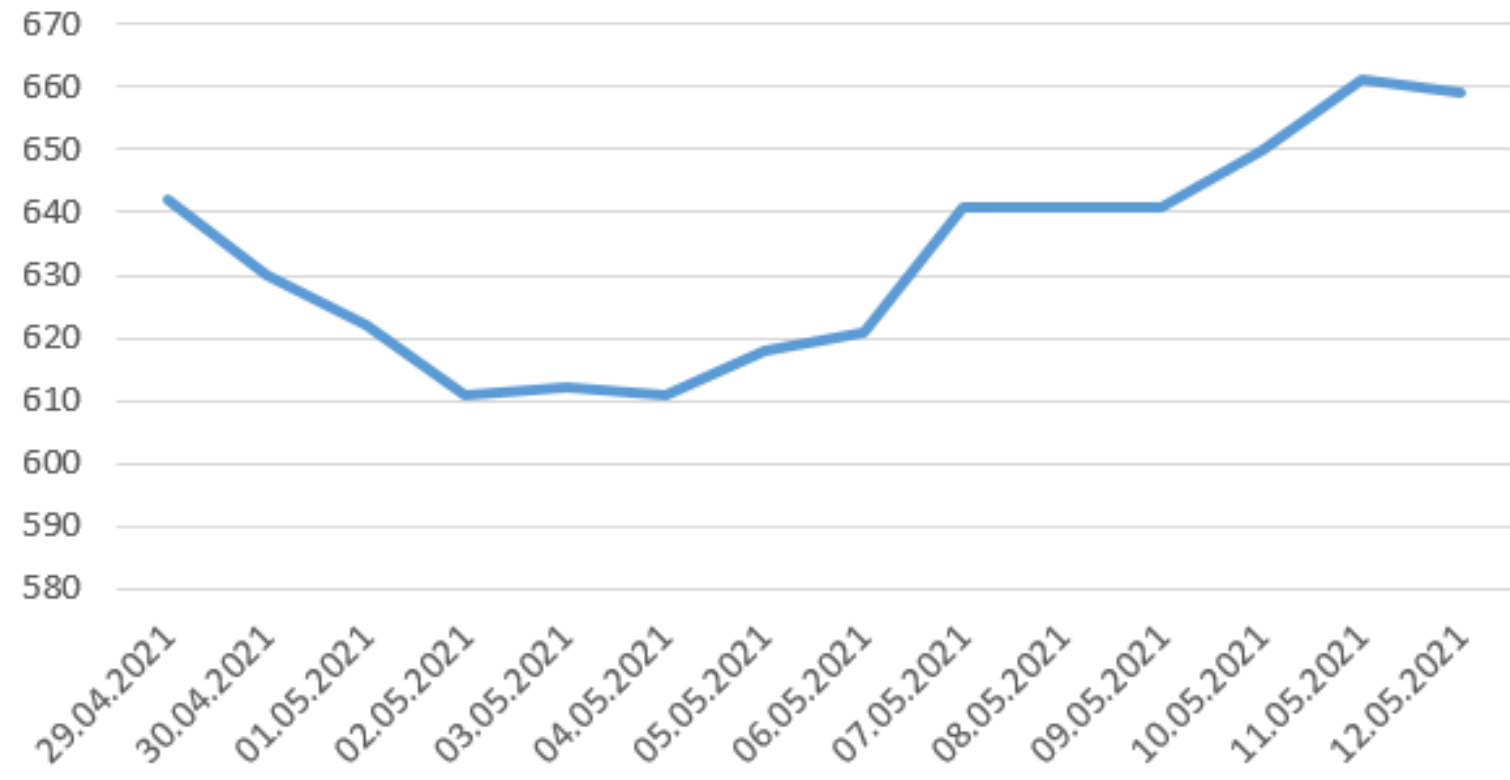

Рисунок 3 - График количества вакансий по запросу "C\# web" 


\section{PHP}

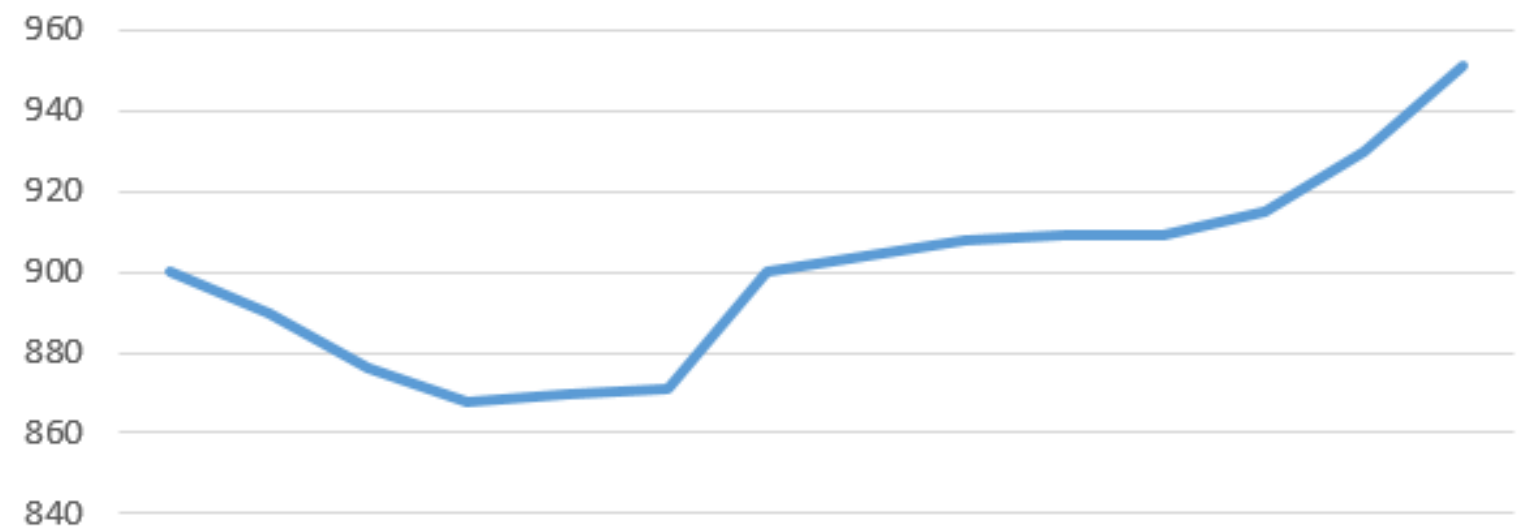

820

Рисунок 4 - График количества вакансий по запросу "Php web"

$$
\text { Node.js }
$$

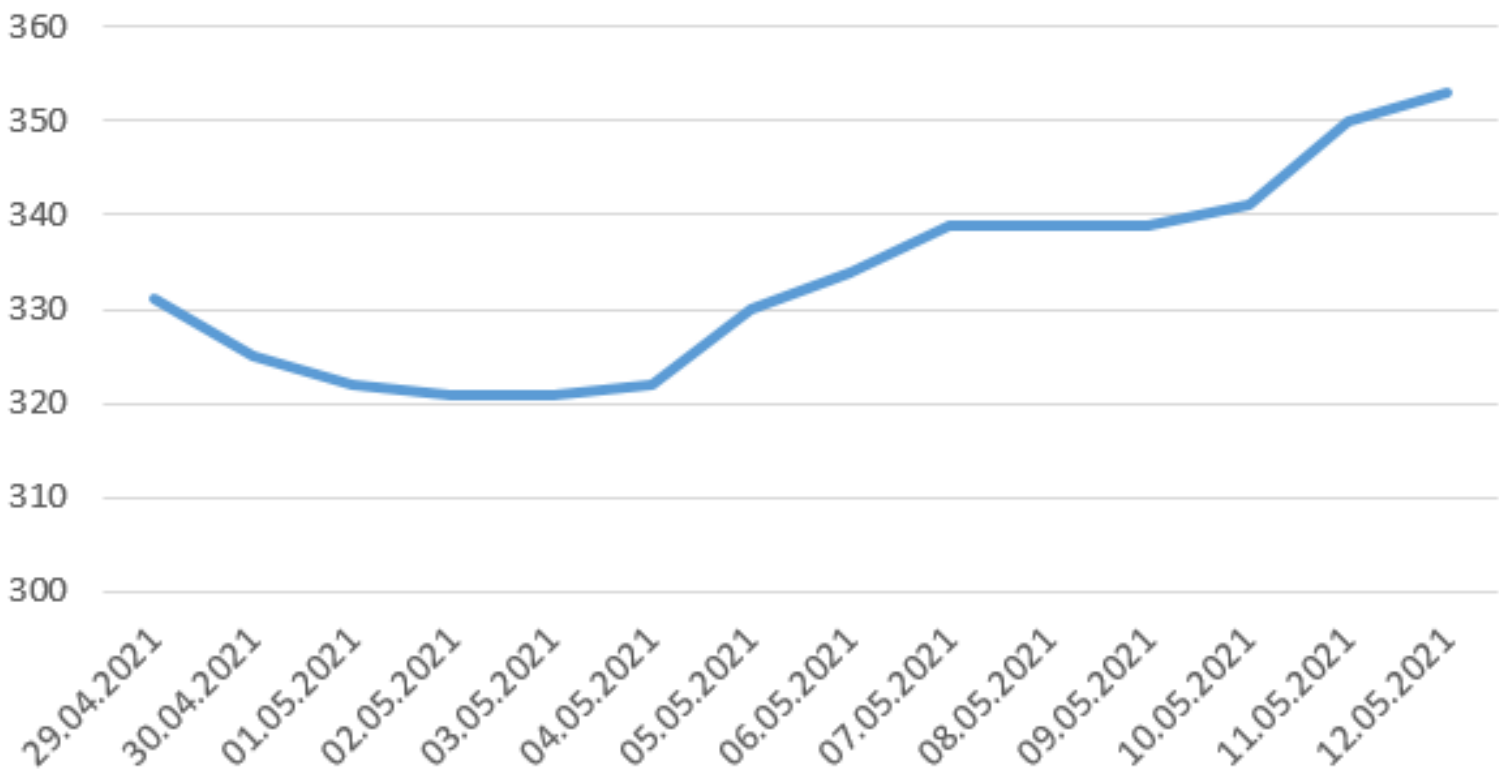

Рисунок 5 - График количества вакансий по запросу "Node.js web" 


\section{Python}

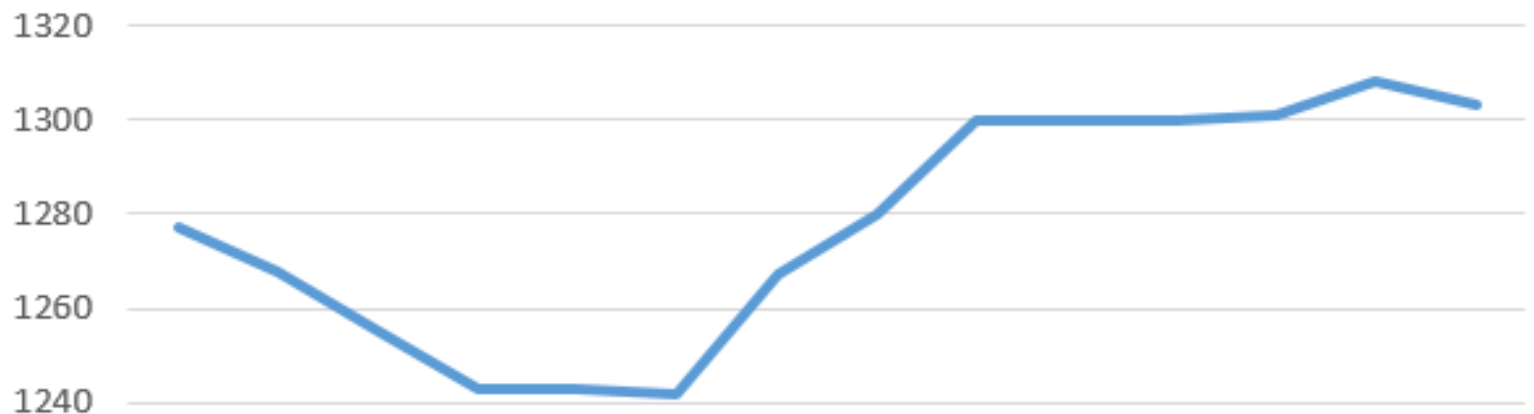

1220

1200

Рисунок 6 - График количества вакансий по запросу "Python web"

Java

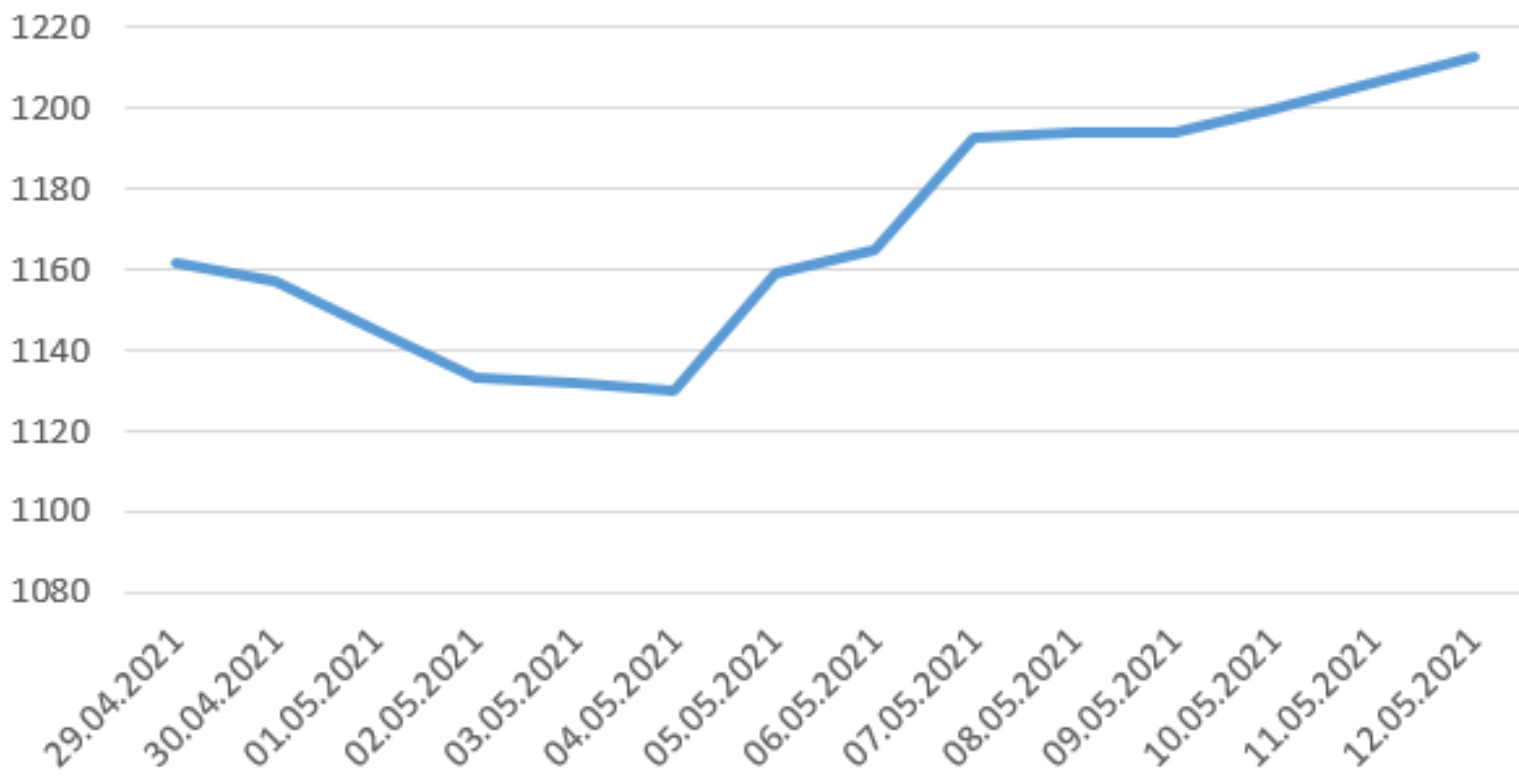

\section{Рисунок 7 - График количества вакансий по запросу "Java web"}

После построения графиков появилась возможность провести анализ числа вакансий, для этого для каждой технологии разработки было найдено среднее арифметическое число, которые использовались для сравнении на следующей гистограмме. 


\section{Технологии разработки}

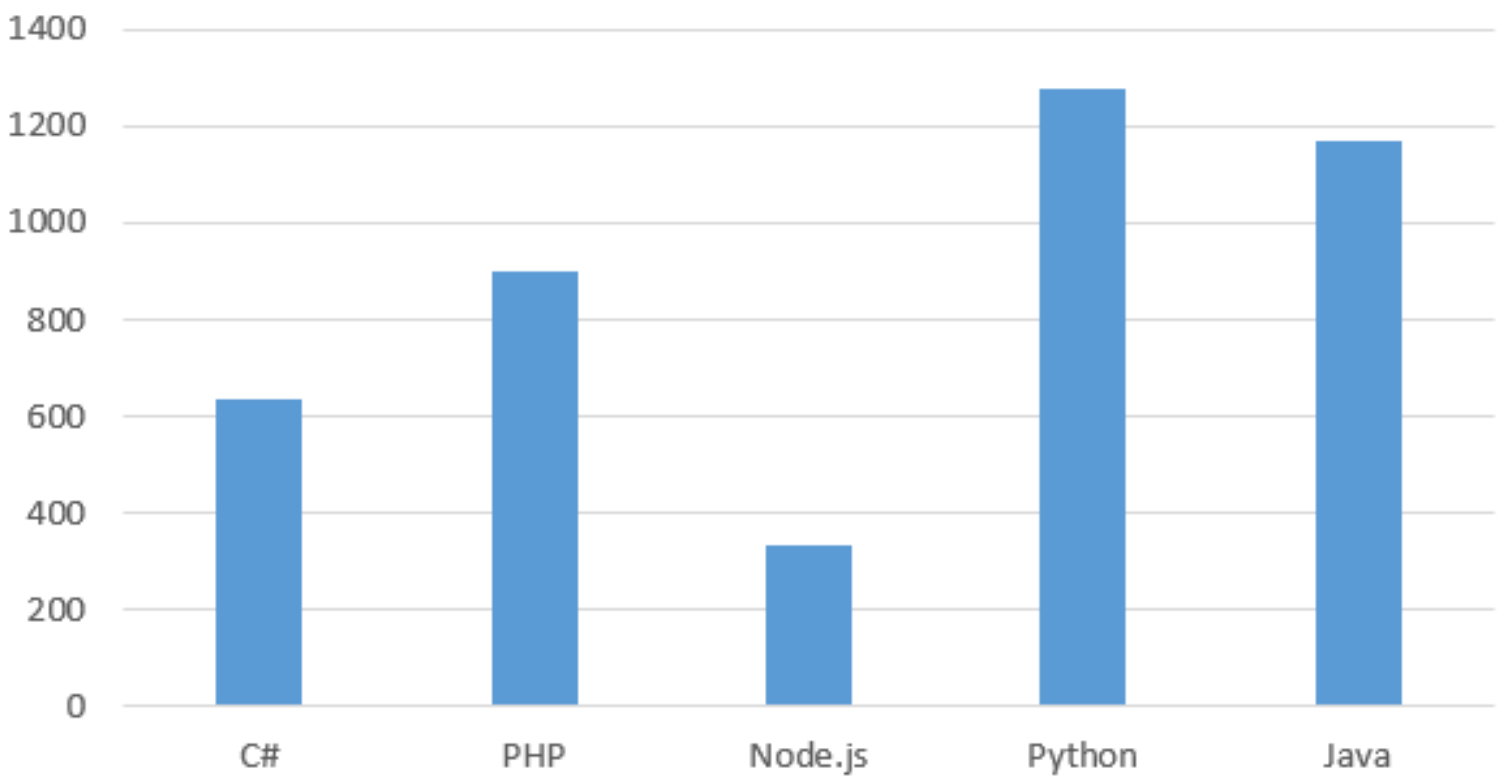

Рисунок 8 - Сравнения числа вакансий технологий разработки

В результате, можно сделать вывод о том, что одними из самых востребуемых технологий на рынке труда являются Python, Java и PHP, которые значительно отрываются от своих конкурентов по числу открытых вакансий в сфере webразработки.

\section{Заключение и выводы}

Полученные результаты позволили сравнить количество вакансий по технологиям разработки и их популярностью. Самыми востребуемыми технологиями разработки серверной части web-сайтов за май стали: Python, Java и PHP. Следует заметить, что в рейтинге PYPL за май они идут в таком же порядке, где Python занимает первое место, Java второе, а РНР четвёртое.

Таким образом, владея этими технологиями можно проще начать карьеру в сфере web-разработки, в том числе и серверной.

Однако, все указанные в статье решении имеют место на мировой практике и используются и развиваются в настоящее время, поскольку популярные web-сайты зачастую не ограничиваются одной технологией, успешно применяя их в тех областях, где они лучше остальных. Примерами таких web-сайтов могут выступать: Amazon, Netflix и Uber.

\section{Литература:}

1. Лекция 1: Основы функционирования веб-приложений. URL: intuit.ru/studies/courses/4455/712/lecture/21291?page=3. Дата обращения (01.05.21).

2. Руководство по Node.js, часть 1: общие сведения и начало работы. // ruvds.com: Компания RuVds. URL: habr.com/ru/company/ruvds/blog/422893/. Дата обращения (27.04.21).

3. Преимущества языка программирования Java // productstar.ru. Url: 
vc.ru/dev/144873-preimushchestva-yazyka-programmirovaniya-java. Дата обращения (19.04.21).

4. Developer Survey 2020 // Web-сайт Stack Overflow Url: insights.stackoverflow.com/survey/2020. Дата обращения (19.04.21).

5. Как работает WEB. Клиент-серверная модель и архитектура вебприложения // Компания Мерион Нетворкс 2021 // Url: zen.yandex.ru/media/merion_networks/kak-rabotaet-web-klientservernaia-model-iarhitektura-vebprilojeniia-604 $\overline{c c} 2 \mathrm{f} 7126 \mathrm{a} 3 \mathrm{~d} 455 \mathrm{aa} 100 \mathrm{dc}$. Дата обращения (20.04.21).

6. Клиентская и серверная веб-разработка. Url: bestprogrammer.ru/programmirovanie-i-razrabotka/klientskaya-i-servernaya-vebrazrabotka. Дата обращения (24.04.21).

7. Введение в серверную часть // платформа MDN Web Docs. Url: developer.mozilla.org/ru/docs/Learn/Server-side/First_steps/Introduction. Дата обращения (24.04.21).

8. 9 самых популярных РНР-фреймворков // ruvds.com: Компания RuVds. Url: habr.com/ru/company/ruvds/blog/539098/. Дата обращения (28.04.21).

9. Библиотека Express // Официальная документация. Url: expressjs.com/ru/. Дата обращения (02.05.21).

10. Основы Java // javahelp.online: web-сайт Striver 2021. Url: javahelp.online/osnovy. Дата обращения (02.05.21).

11. Система поиска вакансий // Url: hh.ru/. Дата обращения (12.05.21).

12. PYPL PopularitY of Programming Language // Url: pypl.github.io/PYPL.html. Дата обращения (13.05.21).

13. TIOBE Index for May 2021 // Url: tiobe.com/tiobe-index/. Дата обращения $(13.05 .21)$.

\section{References.}

1. Lecture 1: Fundamentals of the functioning of web applications. Retrieved May 1, 2021, from intuit.ru/studies/courses/4455/712/lecture/21291?page $=3$.

2. ruvds.com: RuVds Company. Node.js Tutorial Part 1: Getting Started and Getting Started. Retrieved April 27, 2021, from habr.com/ru/company/ruvds/blog/422893/.

3. Website productstar.ru. Advantages of the Java programming language. Retrieved April 19, 2021, from vc.ru/dev/144873-preimushchestva-yazyka-programmirovaniya-java.

4. Website Stack Overflow. Developer Survey 2020. Retrieved April 19, 2021, from insights.stackoverflow.com/survey/2020.

5. Company Merion Networks 2021. How WEB works. Client-server model and web application architecture. Retrieved April 20, 2021, from zen.yandex.ru/media/merion_networks/kak-rabotaet-web-klientservernaia-model-i-arhitekturavebprilojeniia-604cc2f7126a3d455aa100dc.

6. Client and server web development. Retrieved April 24, 2021, from bestprogrammer.ru/programmirovanie-i-razrabotka/klientskaya-i-servernaya-veb-razrabotka.

7. MDN Web Docs platform. Introduction to the server side. Retrieved April 24, 2021, from developer.mozilla.org/ru/docs/Learn/Server-side/First_steps/Introduction.

8. ruvds.com: RuVds Company. 9 most popular PHP frameworks. Retrieved April 28, 2021, from habr.com/ru/company/ruvds/blog/539098/.

9. Official documentation. Express Library. Retrieved May 2, 2021, from expressjs.com/ru/.

10. javahelp.online: Striver 2021 website. Java Basics. Retrieved May 2, 2021, from javahelp.online/osnovy. 
11. Job search system. Retrieved May 12, 2021, from hh.ru/.

12. PYPL PopularitY of Programming Language. Retrieved May 13, 2021, from pypl.github.io/PYPL.html.

13. TIOBE Index for May 2021. Retrieved May 13, 2021, tiobe.com/tiobe-index/.

Abstract. The article describes the main features of creating websites. The main goals and areas of application of client and server development are described. Modern technologies for server development are considered: C \#, PHP, Python, Node.js, Java, as well as the corresponding libraries. The relevance of technologies in the field of web development in the labor market is shown.

Key words: website, server side development tools, programming languages.

Статья отправлена: 15.05.2021 г. (C) Русаков А.М. Семыкин Д.А. 